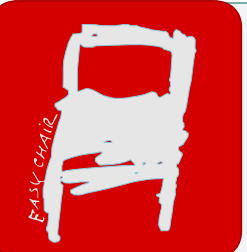

EPiC Series in Health Sciences

Volume 2, 2018, Pages 242-247

\title{
Robot-assisted screw fixation in upper cervical spine using TiRobot system: an accurate and reliable procedure
}

Qiang Yuan $\mathrm{MD}^{1}$, Jingye $\mathrm{Wu}, \mathrm{MD}, \mathrm{FRCSEd}^{2}$, Yajun Liu, MD, FRCSEd ${ }^{3}$, Yonggang Xing, $\mathrm{MD}^{4}$, Yong Zhang ${ }^{5}$, Wei Tian, MD, PhD, FRCSEd ${ }^{6}$

${ }^{1-4,}{ }^{6}$ Department of Spine Surgery, Peking University 4th Clinical Medical College, Beijing Jishuitan Hospital, No. 31, Xinjiekou East Street, Xicheng District, Beijing 100035, People's Republic of China.

${ }^{5}$ TINAVI Medical Technologies Co., Ltd.

${ }^{1}$ First author, email: yuanqiangjst@126.com

${ }^{6}$ Corresponding author, e-mail: tianwei_victor@163.com

\section{INTRODUCTION}

Screw fixation in upper cervical spine surgery is one of the most challenging spinal procedures. Vital anatomical structures, including vertebral arteries, spinal cord and upper cervical nerve roots, are adjacent to the screw trajectories. For that reason, the free-hand techniques are not a reliable procedure in upper cervical spine ${ }^{1}$. As a result, navigation-assisted screw fixation is becoming widely used. Bredow et $\mathrm{al}^{2}$ reported on their use of navigation with good results. However, the limitation of surgeons, such as insufficient steadiness and force control and a steep learning curve, may cause severe problems during procedures. For severe deformity, even when surgeons use navigation to identify the perfect trajectory, screw insertion may still be difficult. Wei Tian ${ }^{3}$ 
reported the first posterior $\mathrm{C} 1-2$ transarticular screw fixation which was assisted by robotic systems using TiRobot system in 2016. There is limited literature describing that procedure since then and large series of robot-assisted screw fixation in upper cervical spine was absent. Our study was aimed to assess the accuracy and reliability of screw fixation in upper cervical spine prospectively.

\section{MATERIALS AND METHODS}

All the patients undergoing screw fixation assisted by robotic system (TiRobot) in upper cervical spine were prospectively studied from August 2015 to January 2018 in Beijing Jishuitan hospital. TiRobot system was co-designed by Beijing Jishuitan hospital and TINAVI Medical Technologies Co., Ltd..

The TiRobot is not a "bone mounted robot" but an image-guided robotic positioning platform, which comprises robotic arm system, optical tracking system and the software workstation. During surgeries, intraoperatively obtained images by C-arm were transferred into the TiRobot system and three-dimensional images were created. Surgeons' plannings of the screw trajectories were performed in TiRobot system. Afterwards, the robot arm with a guidance tube on its end was automatically moved to the entry point of each trajectory and held still. Guiding pins were inserted. A fluoroscopic re-scan by $\mathrm{C}$-arm was performed and followed by cannulated or conventional screws placements.

Clinical accuracy which refers translational and angular deviation, was measured by comparing the guiding pins placement, on reconstructed images obtained by $\mathrm{C}$-arm (called CBCT), to the planned k-wires entry point and trajectory at a depth of $25 \mathrm{~mm}$ in the axial and sagittal planes. To enable a comparison in the same plane, CBCT-toCBCT overlay software (TiRobot Planning Software) was used to fuse each patient's two 3D fluoroscopy scans. In the axial plane, positive translational deviations denote a lateral deflection of the entry point, and positive angular deviations denote a more 
lateral trajectory. In the sagittal plane, positive translational deviations denote a superior deflection of the entry point, and positive angular deviations denote a more cranial trajectory.

\section{RESULTS}

Twenty-six patients (13 males/13 females) were included in this study. The average age was forty-nine. The underlying diseases of these patients covered dens fractures, Hangman's fractures, congenial and acquired deformities. 64 screws were placed assisted by TiRobot system. The synthetic translational deviation was $0.91 \pm 0.37$, the synthetic angular deviation was $2.4 \pm 0.85$ degrees. No screw perforations were detected on postoperative CT scans.
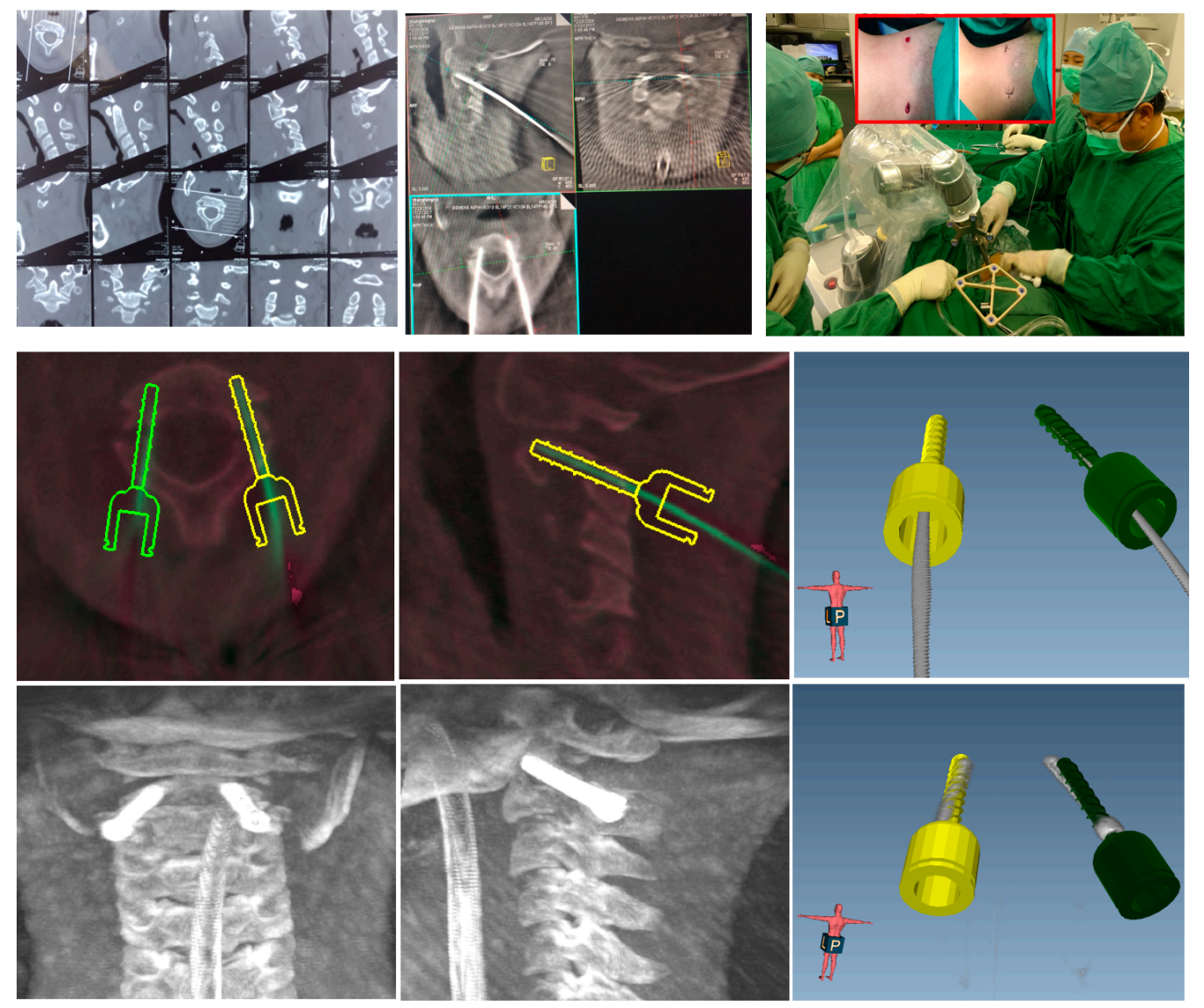
Figure1. 12 years old girl sustained head injury causing Hangman fracture. Two cannulated screws were inserted into the pars interarticularis of $\mathrm{C} 2$ using TiRobot system.

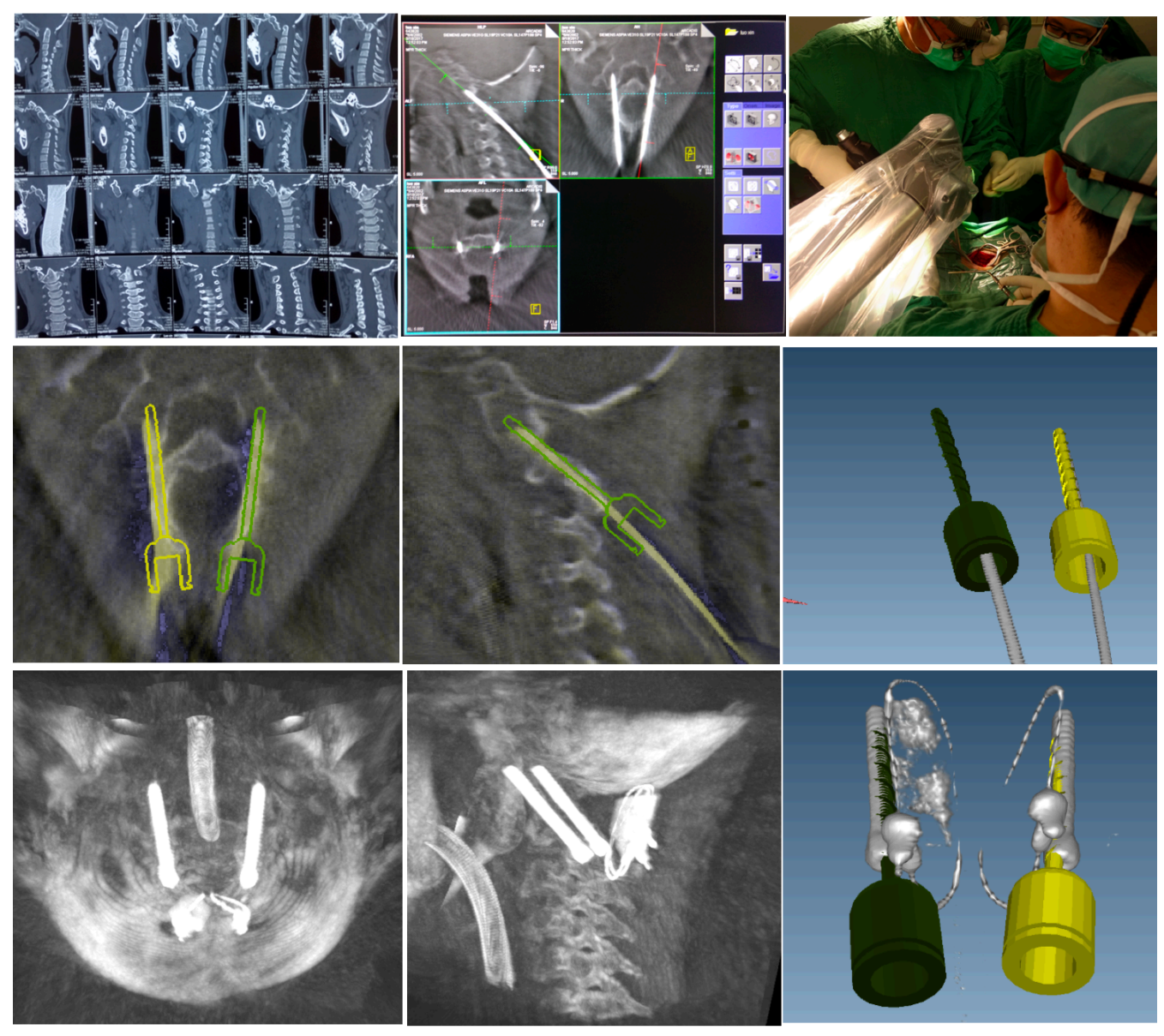

Figure 2. 15 years old girl suffered from atlantoaxial instability and cranial invagination. Margerl screws were inserted using TiRobot system.

\section{DISCUSSIONS}

Kwoh et $\mathrm{al}^{4}$ designed the first neurosurgery robot arm based on PUMA200 in 1985. Mazor Inc. designed a miniature robotic system in 2003 called Spine Assist ${ }^{5}$ Since then, robotic guidance has become an accurate method for pedicle screw place- ment ${ }^{6}$. 
However, most screw placements were only involving the thoracic and lumbar spine. Cervical spine, particularly the upper cervical spine has small size bony structures, higher accuracy requirements are significantly important. The results of our study showed only $0.91 \mathrm{~mm}$ translational deviations with 2.4 degrees angular deviations between planned and real trajectories occurred. This level of accuracy allowed safe and accurate placement of screws in upper cervical spine and no screw perforations were observed. For that reasons, screw fixation in upper cervical spine is an accurate and reliable procedure using TiRobot system.

\section{REFERENCES}

1.Richter M, Cakir B, Schmidt R. Cervical pedicle screws: conventional versus computer-assisted placement of cannulated screws. Spine (Phila Pa 1976) 30: 2280$2287,2005$.

2.Bredow J, Oppermann J, Kraus B, et al. The accuracy of 3D fluoroscopy-navigated screw insertion in the upper and subaxial cervical spine[J]. Eur Spine J 2015;1-10.

3.Tian W. Robot-Assisted Posterior C1-2 Transarticular Screw Fixation for Atlantoaxial Instability: A Case Report. Spine (Phila Pa 1976). 2016 Oct;41 Suppl 19:B2-B5.

4. Kwoh YS, Hou J, Jonckheere EA, et al. A robot with improved absolute positioning accuracy for CT guided stereotactic brain surgery. IEEE Trans Biomed Eng 1988;35:153-60.

5.Shoham M, Burman M, Zehavi E, et al. Bone-mounted miniature robot for surgical procedures: concept and clinical applications. Robotics \& Automation IEEE Transactions on 2003;19: 893-901. 
6. Devito DP, Kaplan L, Dietl R, et al. Clinical acceptance and accuracy assessment of spinal implants guided with Spine Assist surgical robot: retrospective study. Spine 2010;35:2109-15. 